\title{
Effective Design for Optical CDMA Based on Radio over Fiber (RoF) Technique
}

\author{
Rashidi C. B. $\mathrm{M}^{1, *}$, Aljunid S. A ${ }^{1}$, Rahman A.K ${ }^{1}$, Anuar M.S ${ }^{1}$, and Yaakob $\mathrm{S}^{2}$ \\ ${ }^{1}$ Advanced Communication Engineering, Centre of Excellence (ACE-CoE), School of Computer \\ and Communication Engineering, Universiti Malaysia Perlis,Kangar, Perlis, \\ ${ }^{2}$ TM Research and Development, Cyberjaya, Selangor Darul Ehsan, MALAYSIA
}

\begin{abstract}
In this paper, the performance of OCDMA coding systems utilizing the radio over fiber $(\mathrm{RoF})$ technique is presented. It has been done by means of conventional OptiSystem simulation tools, where the propagation of radio signals up to $50 \mathrm{~km}$ using standard single mode fiber (SMF) was investigated. The analysis was made based on the performance of eye diagram, bit rate, bit error rate and optical received power.
\end{abstract}

\section{Introduction}

As the integration of wireless and optical communications, radio-over-fiber (RoF) technology has triggered tremendous interest in being considered as the powerful solution for future high-speed wireless access networks [1]. By using a fiber to connect centralstation (CS) with multiple remote simplified base-stations (BS), it can considerably increase the network capacity, coverage and mobility and furthermore serve both fixed and mobile end users [2]. Recently, many investigations have been done about the RoF system and most of them are mainly focused on the overall architecture design, the RF signal generation or the crosstalk reduction [3-5]. Since security is one of the most important performance indexes of a system, it is of great urgency and significance to improving the security of the RoF system.

Optical code-division multiple access (OCDMA) is a promising multiple-access method for high secure access networks [6]. It combines the large bandwidth of fiber with the flexibility of CDMA technique, so it can achieve high-speed connectivity and guarantee the security against interception along the fiber link. Furthermore, OCDMA also has other attractive features like all optical processing, asynchronous transmission, bandwidth efficiency, soft capacity on demand, protocol transparency, simplified network control and so on [7-8]. In consequence, OCDMA technique can be introduced into the RoF system to form the OCDMA-RoF system and it can solve the security problem of the traditional RoF system.

\footnotetext{
* Corresponding author: rashidibeson@unimap.edu.my
} 


\section{Essential of radio over fiber technique}

RoF presents the distribution of radio frequency (RF) signals over optical fiber links from a central office to RAU. Radio over fiber-scheme for both down and up-link paths are demonstrated based on digitized RF over fiber technique. The digitized RF-over fiber technique is able to improve spectral efficiency and minimize hardware count, while the performance is limited by inter-channel interference as a result of spectra aliasing of the regenerated IF signals which limits the signal-to-noise-ratio [8]. There are many techniques which are used to transport the RF signal to end-users such as; RF-over-fiber, intermediate frequency (IF) over fiber, and baseband (IF) over fiber. The first two former schemes are examples of analog photonic links and the last is a digital photonic link.

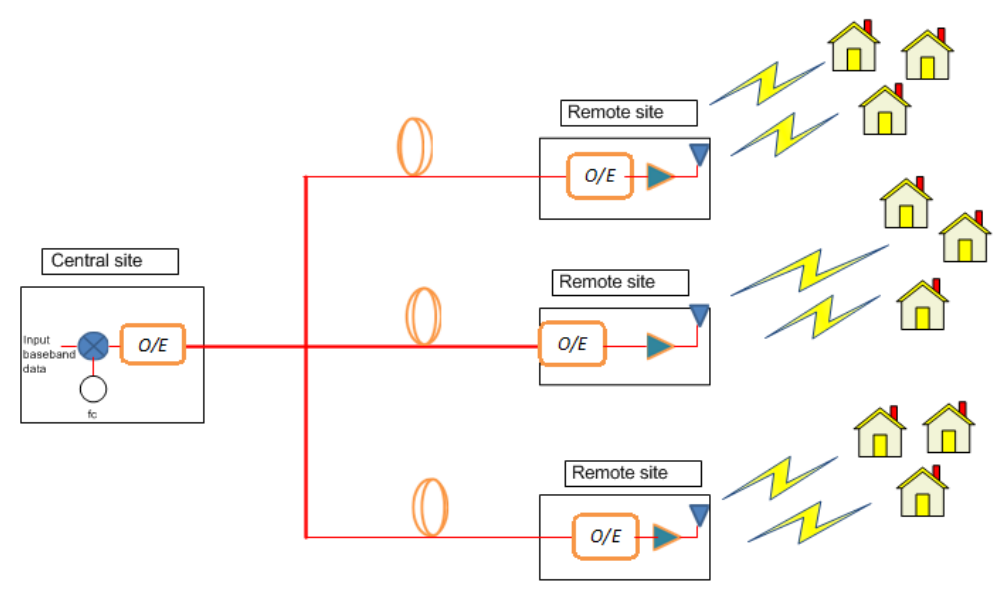

Fig. 1. Basic of RoF Architecture [10]

Figure 1 shows the RF signal is modulated at the central site and combined with continuous wave (CW) laser by external Mach-Zehnder Modulator (MZM), which converts the signal to optical with direct transmission through the fiber to the BSs. At the $\mathrm{BS}$, the signal is detected by the photo-detector (PD) without any need for frequency translation at the remote BS. RF-over fiber transport has the advantage of realizing simple base-station designs with additional benefits of centralized control, independently of the air-interface and also enabling multi wireless band operation. However, one of its major drawbacks is the requirement for high-optical modulation techniques that have the ability to generate speed modulated optical signals as well as high-speed photo-detection schemes that directly convert the modulated optical signals back to signals in the RF domain, Using the external modulator, the dispersion effect can be minimized; thus, this is preferred most in any roof system [9-10].

\section{Optical CDMA coding algorithm}

Optical codes are family of $K$ (for $K$ users) binary $[0,1]$ sequences of length $N$, code weight $W$ (the number of " 1 " in each codeword) and the maximum cross-correlation, $\lambda_{\max }$. In OCDMA system, to allow receivers to distinguish each of the possible users, to reduce channel interference and to accommodate large number of users, optical codes should have large values of $W$ and the size $K$. 
Step 1: The set optical code consists of $\left(N, W, \lambda_{\max }\right)$ FCC code for $K$ users. The $K \times N$ code matrix is here called the Tridiagonal Code Matrix. These sets of codes are then represented by [9];

$$
A_{K}^{W}=\left[\begin{array}{ccccccc}
a_{11} & a_{12} & a_{13} & 0 & 0 & \cdots & 0 \\
a_{21} & a_{22} & a_{23} & a_{24} & 0 & \cdots & \vdots \\
0 & a_{32} & a_{33} & a_{34} & a_{35} & 0 & \vdots \\
0 & 0 & a_{43} & a_{44} & a_{45} & a_{46} & \vdots \\
\vdots & \ddots & \ddots & \ddots & \ddots & \ddots & \vdots \\
0 & 0 & \cdots & \cdots & \cdots & \cdots & a_{K N}
\end{array}\right]=\left[\begin{array}{c}
A_{1} \\
A_{2} \\
A_{3} \\
\vdots \\
\vdots \\
A_{K}
\end{array}\right]
$$

where,

$$
\begin{gathered}
A_{1}=a_{11}, a_{12}, a_{13} \ldots a_{1 N} \\
A_{2}=a_{21}, a_{22}, a_{23}, a_{24} \ldots a_{2 N} \\
A_{3}=a_{31}, a_{32}, a_{33}, a_{34} \ldots a_{3 N} \\
\vdots \\
A_{K}=a_{K 1}, a_{K 2}, a_{K 3} \ldots a_{K N}
\end{gathered}
$$

The rows of $\mathrm{A}_{1}, \mathrm{~A}_{2}$ and $\mathrm{A}_{k}$ represent the $K$ codeword and it is assumed that, the code weight of each of the $K$ codeword is to be $W$.

Step 2: After the $K$ codes, represented by the $K$ rows of the $K \times N$ code matrix $A_{K}^{W}$ in Eq. (1), are to represent a valid set of $K$ codeword with in phase cross-correlations $\lambda_{\max }$ and code weight $W$; it must satisfy the following conditions;

1. The elements $\left\{\mathrm{a}_{\mathrm{ij}}\right\}$ of $A_{K}^{W}$ must have values " 0 " or " 1 "

$$
\mathrm{a}_{\mathrm{ij}}=\text { " } 0 \text { " or " } 1 \text { " for } \mathrm{i}=1,2, . . K, \mathrm{j}=12, . . N
$$

2. The in phase cross-correlation $\lambda_{\max }$, between any of the $K$ code words ( $K$ rows of the matrix $A_{K}^{W}$ ) should not exceed code weight $W$. That is,

$$
X_{i} X_{j}^{T}= \begin{cases}\leq \lambda_{\max } & \text { for } i \neq j \\ =W & \text { for } i=j\end{cases}
$$

3. The code weight of each codeword should be equal to $W$ where,

$$
\sum_{j=1}^{N} a_{i j}=W, i=1,2 \ldots K
$$

4. From Eq. (3), it is seen that the $W=X_{\mathrm{i}} X_{\mathrm{i}}^{\mathrm{T}}$ is the in phase auto-correlation function of codes. $X_{\mathrm{i}} Y_{\mathrm{j}}^{\mathrm{T}}$ is the out of phase cross-correlation between the $i^{\text {th }}$ and the $j^{\text {th }}$ codes. It follows that $X_{\mathrm{i}} X_{\mathrm{i}}^{\mathrm{T}}$ should be greater than $X_{\mathrm{i}} Y_{\mathrm{j}}^{\mathrm{T}}$. In other words, $W>\lambda_{\max }$.

5. All $K$ rows of $A_{K}^{W}$ should be linearly independent because each codeword must be uniquely different from other code words. That is to say the rank of the $A_{K}^{W}$ code matrix should be $K$. Moreover, for $A_{K}^{W}$ to have rank $K$, thus codes $N \geq K$.

Step 3: From the five conditions above in Step 2, one of the matrices binary sequences as shown in Eq. (1), whose the first $i^{\text {th }}$ row for the first $K$ user is given by;

$$
A_{i}=\begin{array}{ccc}
r(i-1) & W & r(K-i) \\
0 \ldots \mathrm{O} & 11 \ldots 1 & 0 \ldots \mathrm{O}
\end{array}
$$


The length $N$ of the codes which is the length of the rows of the $K \times N$ code matrix is given by;

$$
N=W K-\lambda_{\max }(K-1)
$$

It can be seen that the length $\mathrm{N}$ is minimum under the assumed conditions.

\section{Result and discussion}

The performance of OCDMA-RoF was demonstrated by using OptiSystem software from Optiwave ${ }^{T M}$ and the simulation parameters are shown in Table 1. The simulation parameters such as attenuation, dispersion effects and non-linear effects such as four wave mixing are activated during simulation and specified according to the typical industry values to simulate the real environment as close as possible. The performances of the system were characterized by referring to the fiber length and effective optical received power, ORP for the OCDMA-RoF.

Table 1. Typical parameters for the simulation analysis.

\begin{tabular}{|c|c|}
\hline Parameters & Values \\
\hline Data rates & $155,622 \mathrm{Mbps}$ \\
\hline Operating wavelength & $\lambda=1550 \mathrm{~nm}$ \\
\hline Number of users, $K$ & 3,9 \\
\hline Number of code weights, $W$ & 2,4 \\
\hline Attenuation & $0.2 \mathrm{db} / \mathrm{km}$ \\
\hline Thermal noise & $1.0 \times 10^{-22} \mathrm{~W} / \mathrm{Hz}$ \\
\hline Dispersion & $16.75 \mathrm{ps} / \mathrm{nm}-\mathrm{km}$ \\
\hline Fiber length (km) & $10-80$ \\
\hline Fiber loss & $0.2 \mathrm{~dB}$ \\
\hline Pulse width & $0.8 \mathrm{~nm}$ \\
\hline
\end{tabular}

Fig 2 shows the plots between fiber length and system performance BER for OCDMARoF for bit rates of $155 \mathrm{Mbps}$ and $622 \mathrm{Mbps}$ when the number of users fixed at nine, $(K=9)$. It can be seen that, at system performance BER of $10^{-9}$, bit rate of $155 \mathrm{Mbps}$, the OCDMA-RoF code definitely has better system performance BER where it is capable of achieving an acceptable BER threshold adequately for $45 \mathrm{~km}$ fiber length. In contrast the OCDMA-RoF code can perform adequately for $10 \mathrm{~km}$ fiber length within bit rate of 622 Mbps and system performance BER of $10^{-9}$. It is clearly observed that, from the eye diagrams bit rate of $155 \mathrm{Mbps}$ having a large eye-opening between the top and bottom level. The height of the eye-opening at the specified time integral shows the noise margin or immunity to noise. While, at a bit rate of $622 \mathrm{Mbps}$ shows the more the eye close the more difficult it is to differentiate between the ones and zeros in the signal. From this observation, OCDMA-RoF at bit rate of $155 \mathrm{Mbps}$ can successfully eliminate and suppressed the effects of PIIN and MAI for the SAC-OCDMA coding system. 


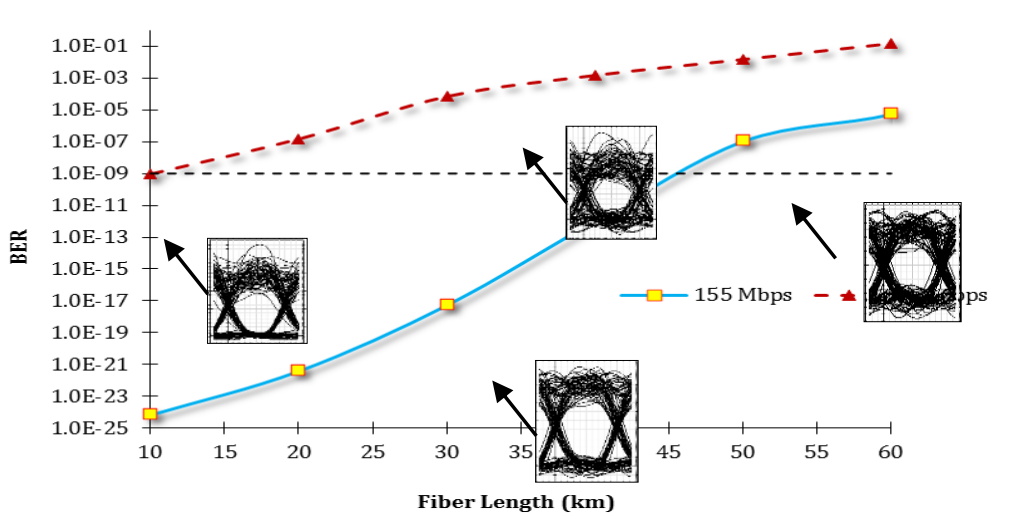

Fig. 2. Effect of bit rates on fiber length for OCDMA-RoF.

Fig 3 shows the plots of optical received power, ORP versus system performance BER for OCDMA-RoF based on AND subtraction detection technique for OCDMA coding system. This evaluation is to see the effect of bit rates of $155 \mathrm{Mbps}, 622 \mathrm{Mbps}$ and $1 \mathrm{Gbps}$, respectively on ORP. From this figure, at a bit rate of $155 \mathrm{Mbps}$, the OCDMA-RoF coding system can achieve an optimum value of optical received power ORP $=-25 \mathrm{dBm}$ without requiring any amplification. At system performance BER of $10^{-9}$, while at at bit rates of $622 \mathrm{Mbps}$ and 1 Gbps can accommodate minimum optical received power ORP $=-19 \mathrm{dBm}$ and $-18 \mathrm{dBm}$, respectively. There are about $-36 \mathrm{~dB}$ margin between bit rate of $155 \mathrm{Mbps}$ and $622 \mathrm{Mbps}$, where - $37 \mathrm{~dB}$ between bit rate of $155 \mathrm{Mbps}$ and $1 \mathrm{Gbps}$ effective received power margins for optical transmission.

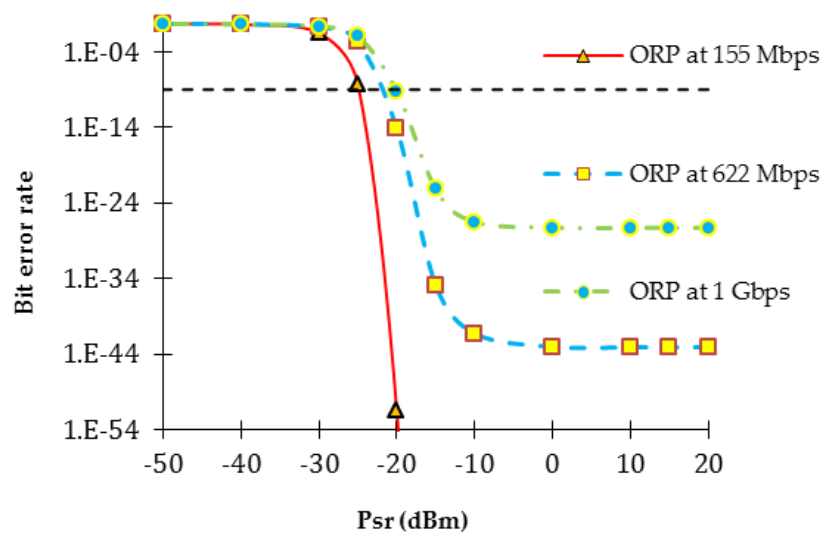

Fig. 3. Effect of various bit rates on optical received power (ORP).

\section{Summary}

The performances of the OCDMA coding systems using Radio over Fiber (RoF) have been presented. The results indicated that the OCDMA-RoF achieves long transmission up to $50 \mathrm{~km}$ at minimum effective optical received power ORP $=-25 \mathrm{dBm}$. In addition to that, the architecture of OCDMARoF also presented with a MAI cancellation property. Without a doubt, the maximum out of phase auto-correlation and flexibility in phase cross-correlation values, respectively with different combination of $\left(N, W, \lambda_{\max }\right)$; by increasing $W$ and increasing $\lambda_{\max }$ will improve the system performance with shorter, $N$. This will give an offer in OCDMA-RoF for better quality of service (QoS), fast asynchronous transmission, variable bit rate traffic and higher bandwidth in optical access networks. 


\section{References}

1. Z. Jia, J. Yu, G. Chang, IEEE Photon. Technol. Lett., 18(16), 1726 (2006)

2. Y. Hsueh, Z. Jia, H. Chien, A. Chowdhury, J. Yu, G.K. Chang, J. Lightw. Technol., 29(8), 1105 (2011)

3. D. Wake, A. Nkansah, J. Gomes, J. Lightw. Technol., 28(16), 2456 (2010)

4. Abdullah O. Aldhaibania, S. Yaakobb, R.Q. Shaddada, S.M. Idrusa, M.Z. Abdul Kadirb, A.B. Mohammada, Optik, 3678 (2013)

5. S. Yaakob, N. M. Samsuri, R. Mohamad, N.E. Farid, I.M. Azmi, S.M. Mohd. Hassan, N. Khushairi, S.A. Enche Ab Rahim, A.I. A Rahim, A. Rasmi, A.K. Zamzuri, S.M. Idrus, S.H. Fan, The 3rd International Conference on Photonics, (2012)

6. S. Yaakob, M.A. Ismail, R. Mohamad, M.R. Yahya, A.F.A. Mat, M.R. Mokhtar, H.A.A Rashid, IEEE International Conference On Semiconductor Electronics, (2006)

7. C. Zhang, L. Wang, K. Qiu, Opt. Express, 19(15), 13957 (2011)

8. B. Zhang, Y. Lu, J. Zhang, B. Yang, (Book Chapter FKE, UTM, 2007)

9. B. Hraimel, X. Zhang, Y. Shen, International Conference in Microwave and Millimeter Wave Technology (ICMMT), (2012)

10. C.B.M. Rashidi, S.A. Aljunid, F. Ghani, H.A. Fadhil, M.S. Anuar, Procedia Eng., 53, $420(2013)$

11. S.M. Idrus, I.N. Salleh, N.S. Sahedan, S.Y. Cheng, V.O.S. Yee, (Book Chapter FKE, UTM, 2008) 